\title{
Author Correction: The new nanophysiology: regulation of ionic flow in neuronal subcompartments
}

David Holcman and Rafael Yuste

Nature Reviews Neuroscience (2015) https://doi.org/10.1038/nrn4022

Published online 14 October 2015

In this article, the origin of some of the presented modelling information should have been stated and insufficient details were provided to understand how the curve in Box 2 and the electrodiffusion-related curves in Figure 3 were generated.

Equation 5 in Box 2 and some of the simulated data in Figure 3 came from the following study: Schuss, Z., Cartailler, J. \& Holcman, D. Poisson-Nernst-Planck equations in a ball. Preprint at $\operatorname{arXiv}$ https://arxiv.org/abs/1505.02173 (2015) (later published as: Cartailler, J., Schuss, Z. $\&$ Holcman, D. Analysis of the Poisson-Nernst-Planck equation in a ball for modelling the voltage-current relation in neurobiological microdomains. Physica D 339, 39-48 (2017)).

Equation 5 is a simplified version of equation 9 in Schuss et al., but it was not made clear that it had been simplified, and the simplified version made it difficult to understand how the aforementioned curves were generated. Equation 5 should have been written in the form

$$
-\Delta \varphi=\frac{Q e^{-\frac{z e \varphi}{k T}}}{\varepsilon \varepsilon_{0} \int_{\Omega} e^{-\frac{z e \varphi}{k T}}}
$$

for which the compatibility condition at the boundary is

$$
\frac{\partial \varphi}{\partial n}=-\frac{Q}{\varepsilon \varepsilon_{0}|\partial \Omega|}
$$

where

$$
|\partial \Omega|=4 \pi R^{2}
$$

for a ball of radius $R$. $R^{2}$ had been omitted in the simplified equation as $R$ was equal to one in this instance. The value of the dielectric constant $(\varepsilon)$, which should have been provided, is 80 .

Parts a-c in Figure 3 show concentration of ions against the radius of the sphere. Here, the concentrations values were calculated from the density of particles (number of ions per $\mu \mathrm{m}^{3}$ ), which was given by

$$
\rho(r)=\frac{Q e^{-\frac{z e \varphi(r)}{k T}}}{z e \int_{\Omega} e^{-\frac{z e \varphi}{k T}} d x}
$$

where the voltage potential $\varphi$ is the solution of equation 5.

https://doi.org/10.1038/s41583-019-0182-9 | Published online 23 May 2019 